\title{
Possible implication of vagal nerve stimulation for treating refractory psoriasis
}

\section{Possível implicação da estimulação do nervo vagal para o tratamento da psoríase refratária}

\author{
Nima Derakhshan', Mahboubeh Kazemi" \\ Shiraz University of Medical Sciences, Shiraz, Iran
}

'MD. Resident in Neurosurgery, Neuroscience Research Center, Shiraz University of Medical Sciences, Shiraz, Iran.

"MD. Obstetrician and Gynecologist, Department of Gynecology and Obstetrics, Shiraz University of Medical Sciences, Shiraz, Iran.
Psoriasis is a chronic, relapsing, immune-based skin disease that affects an estimated 1-3\% of the world's population. ${ }^{1}$ There is no consensus regarding the exact etiology of psoriasis; among several postulated pathophysiological causes, excessive activity of $\mathrm{T}$ cell-mediated immune response, and T-helper (Th) in particular, is the most accepted theory. This is supported by increased levels of pro-inflammatory cytokines, especially tumor necrosis factor (TNF- $\alpha$ ) in the serum, skin lesions and joints of these patients. ${ }^{2,3}$

Although inhibitors of tumor necrosis factor (TNF- $\alpha$ ) have been shown to be effective for treating refractory psoriasis, ${ }^{3,4}$ there are no therapies providing long-lasting remission for these patients.

Since Tracey ${ }^{5}$ proposed the so-called inflammatory reflex, the data now emerging has been elucidating and supporting the existence of a neural circuit that modulates immune response. This cholinergic anti-inflammatory pathway originates from efferent vagal fibers. It seems that inhibition of TNF- $\alpha$ production in the spleen following vagal nerve stimulation occurs through acetylcholine signaling via the $\alpha 7$ nicotinic acetylcholine receptor that is expressed on cytokineproducing macrophages. ${ }^{5,6}$ This signal is relayed through an acetylcholine-producing, memory phenotype $\mathrm{T}$ cell population that has been identified in mice, which is necessary for inhibition of cytokine production through vagus nerve stimulation. ${ }^{7}$

Immunomodulation via vagal nerve stimulation has been implicated in the treatment of other immune disorders involving the TNF- $\alpha$ pathway, such as inflammatory bowel disease. ${ }^{8}$ In this regard, we support the hypothesis that vagal nerve stimulation may prove useful for treating refractory psoriasis and psoriatic arthritis through its cholinergic anti-inflammatory effects, by means of modulating TNF- $\alpha$ production. ${ }^{9}$

This novel idea encourages interest in conducting a double-blind case-control study to investigate the possible role of vagal nerve stimulation in treating psoriasis and psoriatic arthritis. 


\section{REFERENCES}

1. Derakhshan N. NFkB inhibitors as a potential novel hypothesized treatment for psoriasis. Sao Paulo Med J. 2011;129(6):433-4.

2. Zaba LC, Cardinale I, Gilleaudeau P, et al. Amelioration of epidermal hyperplasia by TNF inhibition is associated with reduced Th17 responses. J Exp Med. 2007;204(13):3183-94.

3. Tobin AM, Kirby B. TNF alpha inhibitors in the treatment of psoriasis and psoriatic arthritis. BioDrugs. 2005;19(1):47-57.

4. Kircik LH, Del Rosso JQ. Anti-TNF agents for the treatment of psoriasis. J Drugs Dermatol. 2009;8(6):546-59.

5. Tracey KJ. Reflex control of immunity. Nat Rev Immunol. 2009;9(6):418-28.

6. Pavlov VA, Tracey KJ. The cholinergic anti-inflammatory pathway. Brain Behav Immun. 2005;19(6):493-9.

7. Rosas-Ballina M, Olofsson PS, Ochani $M$, et al. Acetylcholinesynthesizing $T$ cells relay neural signals in a vagus nerve circuit. Science. 2011:334(6052):98-101.

8. Meregnani J, Clarençon D, Vivier M, et al. Anti-inflammatory effect of vagus nerve stimulation in a rat model of inflammatory bowel disease. Auton Neurosci. 2011;160(1-2):82-9.

9. Das UN. Can vagus nerve stimulation halt or ameliorate rheumatoid arthritis and lupus? Lipids Health Dis. 2011;10:19.

\section{Sources of funding: None}

Conflict of interest: None

Date of first submission: February 17, 2015

Last received: February 22, 2015

Accepted: March 11, 2015

\section{Address for correspondence:}

Nima Derakhshan, M.D

Resident of Neurosurgery

Neuroscience Research Center, Department of Neurosurgery,

Shiraz University of Medical Sciences

Chamran Hospital — Chamran Avenue — Shiraz — Iran

Postal Code: 7194815644

Tel/Fax. + 98-711-6234508

Cellphone. +98-917-7161290

Email:nima_med83@yahoo.com 